\title{
Identification of deleterious and regulatory genomic variations in known asthma loci
}

\author{
Matthew D. C. Neville' ${ }^{1}$ Jihoon Choi ${ }^{1}$, Jonathan Lieberman ${ }^{1}$ and Qing Ling Duan ${ }^{1,2^{*}}$
}

\begin{abstract}
Background: Candidate gene and genome-wide association studies have identified hundreds of asthma risk loci. The majority of associated variants, however, are not known to have any biological function and are believed to represent markers rather than true causative mutations. We hypothesized that many of these associated markers are in linkage disequilibrium (LD) with the elusive causative variants.

Methods: We compiled a comprehensive list of 449 asthma-associated variants previously reported in candidate gene and genome-wide association studies. Next, we identified all sequence variants located within the 305 unique genes using whole-genome sequencing data from the 1000 Genomes Project. Then, we calculated the LD between known asthma variants and the sequence variants within each gene. LD variants identified were then annotated to determine those that are potentially deleterious and/or functional (i.e. coding or regulatory effects on the encoded transcript or protein).

Results: We identified 10,130 variants in LD $\left(r^{2}>0.6\right)$ with known asthma variants. Annotations of these LD variants revealed that several have potentially deleterious effects including frameshift, alternate splice site, stop-lost, and missense. Moreover, 24 of the LD variants have been reported to regulate gene expression as expression quantitative trait loci (eQTLS).

Conclusions: This study is proof of concept that many of the genetic loci previously associated with complex diseases such as asthma are not causative but represent markers of disease, which are in LD with the elusive causative variants. We hereby report a number of potentially deleterious and regulatory variants that are in LD with the reported asthma loci. These reported LD variants could account for the original association signals with asthma and represent the true causative mutations at these loci.
\end{abstract}

Keywords: Asthma, Heritability, Linkage disequilibrium, Causal variants, SNPs, eQTLs

\section{Background}

Asthma is a chronic respiratory disease characterized by hyper-responsiveness of the bronchial muscles, inflammation, and reversible narrowing of the airways. It affects over 330 million individuals worldwide and is expected to increase to approximately 400 million by 2025 [1, 2]. Asthma is known to be multifactorial and polygenic in nature with numerous genetic and environmental risk factors. Twin studies have estimated that genetic factors contribute approximately $55-95 \%$ of asthma risk, known as its heritability [3-5]. To date, hundreds of genetic variants have

\footnotetext{
* Correspondence: qingling.duan@queensu.ca

'Department of Biomedical and Molecular Sciences, Queen's University, Botterell Hall, Room 530 - 18 Stuart St, Kingston, ON K7L3N6, Canada ${ }^{2}$ School of Computing, Queen's University, 557 Goodwin Hall, Room 531, Kingston, ON K7L 2N8, Canada
}

(c) The Author(s). 2018 Open Access This article is distributed under the terms of the Creative Commons Attribution 4.0 International License (http://creativecommons.org/licenses/by/4.0/), which permits unrestricted use, distribution, and reproduction in any medium, provided you give appropriate credit to the original author(s) and the source, provide a link to the Creative Commons license, and indicate if changes were made. The Creative Commons Public Domain Dedication waiver (http://creativecommons.org/publicdomain/zero/1.0/) applies to the data made available in this article, unless otherwise stated.

been correlated with asthma and asthma related traits such as lung function and bronchial hyper-responsiveness. However, only a small portion of asthma risk is explained by known genetic loci. For example, in a recent study of 31 genetic variants previously associated for asthma across multiple populations, Vicente et al. determined that these collectively account for only approximately $2.6 \%$ of asthma heritability in the UK Biobank [6]. Thus, our understanding of the genetic determinants of asthma remains limited and further investigations to identify the missing heritability are necessary to facilitate better prevention, diagnosis and care of this chronic disease.

Several factors may account of the missing heritability of asthma such as epistasis, gene-environment interactions, rare variants, and epigenomics (i.e. non-sequence variations 
in the genome) [7]. In addition, the genetic variants previously correlated with asthma may not represent the true causative mutation. Instead, it is widely accepted that the majority of loci identified from genome-wide association studies (GWAS) as well as candidate gene studies are not causal but are genetic markers that tag causal variants [8]. This is supported by the fact that the majority of variants associated with complex diseases such as asthma have no known impact on the resulting transcripts or proteins, with over $80 \%$ from GWAS falling outside of protein coding regions [9]. Discovery of the causal variants underlying a disease would reveal the true genetic effect sizes [8] and help to facilitate the development of more accurate clinical tests for diagnosis and treatment of asthma [10].

In this study, we hypothesize that the causal mutations at numerous asthma-associated loci are likely in linkage disequilibrium (LD) with the associated markers, which refers to the non-random association of alleles at different loci [11]. We test this through the analysis of existing whole-genome sequencing data from large populations to identify genetic variants that are more likely to be causal and explain for the missing heritability of asthma. First, we compiled a comprehensive list of asthma-associated variants from earlier GWAS and candidate gene studies. Next, we identified all variants within these asthma loci using sequence data from the 1000 Genomes Project (1000GP) [12] and calculated LD between the asthma variants and the sequence variants. Then, both asthma variants and the LD variants were annotated for their predicted effects on the resulting transcript or protein. We hereby report a list of potentially deleterious and regulatory variants within known asthma loci that are in LD with and could account for the reported association signals. These results may improve our understanding of the underlying mechanisms of asthma, which will ultimately lead to better prevention and more efficient therapies.

\section{Methods}

\section{Selection of asthma loci}

Previously associated SNPs from asthma GWAS were obtained from the National Human Genome Research Institute-European Bioinformatics Institute (NHGRI-EBI) GWAS Catalog (October 10th, 2017 release) [13]. This manually curated GWAS catalog lists SNP-trait associations with $p$-values $<1.0 \times 10^{-5}$ in studies assaying at least 100,000 SNPs. We selected all SNPs linked to asthma and asthma severity traits: 'Asthma, 'Adult asthma, 'Asthma (childhood onset), 'Asthma (sex interaction),' 'Asthma and hay fever', 'Asthma or chronic obstructive pulmonary disease,' 'Bronchial hyperresponsiveness in asthma', and 'Pulmonary function in asthmatics'.

A compilation of candidate genes were also selected from four previously published lists of asthma candidate gene studies [14-17]. A total of 148 genes that did not overlap with the asthma GWAS loci above were selected. We then proceeded to search for these candidate genes in PubMed using keywords including "asthma", "polymorphism" or "snp", and the gene name. All identified English language candidate gene studies reporting a genetic association for asthma or asthma severity published up to June 2016 were examined. Asthma-associated SNPs reported to a gene on our candidate gene list that were significant at the level defined by the authors of the study were included for further analysis.

\section{Genomic coordinates and 1000 genomes project}

Starting with a comprehensive list of SNPs previously associated with asthma and related phenotypes from GWAS and candidate gene studies, we removed pseudogenes and uncharacterized loci unknown to the RefSeqGene database [18]. Next, the University of Santa Cruz (UCSC) Genome Browser [19] was used to identify the genomic coordinates of each gene using GRCh37/hg19 assembly of the human reference genome. The coordinates of each gene were extended by $5000 \mathrm{bp}$ both $5^{\prime}$ and $3^{\prime}$ of the transcription start sites (TSS) and transcription end sites (TES) of each gene to include potential regulatory regions. These genomic coordinates were then applied to extract a complete list of sequence variations from the 1000GP Phase 3 whole genome sequencing data [12].

\section{Linkage disequilibrium analysis}

At each locus, we calculated the LD between the asthma-associated SNP (reported by GWAS and candidate gene studies) and the newly identified variants from the 1000GP using Plink, version 1.9 [20]. A distance window of $1 \mathrm{Mb}$ was used to determine $\mathrm{LD}$, which excludes variants greater than $1 \mathrm{Mb}$ apart. The $\mathrm{r}^{2}$ threshold for LD was set to $\geq 0.6$.

\section{Functional annotation}

The genomic variant annotations and functional effect prediction toolbox known as SnpEff (version 4.3q) [21] was used with the GRCh37.75 assembly to predict the effects of sequence variants identified from the 1000GP. To validate these annotations, we also used Ensembl's Variant Effect Predictor (VEP), release 90, [22] and only concordant annotations between the two prediction tools were reported. For those variants with multiple annotations from each annotation tool (e.g. variants in regions impacted by multiple transcripts), only the most severe effect as ranked by SnpEff was selected. VEP was also used to access the pathogenicity predictions of missense mutations from the scoring tools Sorting Intolerant From Tolerant (SIFT) [23] and Polyphen-2 [24]. The Database of Genomic Structural Variations (dbVar) [25] was used to supplement annotations for structural variants. 
RegulomeDB (version 1.1) [26] was used to evaluate the potential of variants to impact gene expression. RegulomeDB scores variants for their predicted functional impact based on high-throughput data from non-coding and intergenic regions of the human genome. It uses experimental data sets from the Encyclopedia of DNA Elements (ENCODE) project [27], public datasets from Gene Expression Omnibus (GEO) [28], and curated published literature.

\section{Results}

\section{Asthma loci}

We identified a total of 449 asthma-associated SNPs from earlier GWAS and candidate gene studies. Of these, 225 SNPs were found from asthma GWAS via the NHGRI-EBI GWAS Catalog [13] and mapped to 224 unique genes (Additional file 1: Table S1). In addition, we identified loci reported by candidate gene studies, of which 224 SNPs did not overlap with GWAS loci and mapped to 81 unique genes (Additional file 1: Table S2). Functional annotation of the 449 asthma variants revealed that only $16 \%$ are found in protein coding regions, and fewer still (12\%) are predicted to cause protein coding changes (Additional file 1: Table S3). The majority of associated variants are intronic $(30 \%)$, intergenic $(15 \%)$, or upstream/downstream $(32 \%)$ gene variants (Fig. 1).

\section{Linkage disequilibrium variants}

Using whole-genome sequencing data within the genomic coordinates of the 304 unique asthma genes from GWAS and candidate gene studies, we identified potentially functional variants that are in LD with asthma-associated variants. Specifically, we extracted a total of 1,385,534 variants from these 305 loci using Phase 3 sequence data of the 1000GP. We then assessed LD between the asthma variants and the sequence variants from the 1000GP using data from each of the five continental ancestry groups (African, American, East Asian, European, and South Asian) to account for the discordant nature of LD among ethnic groups $[29,30]$. All variants in $\operatorname{LD}\left(r^{2} \geq 0.6\right)$ in one or more of the five ancestry groups were included for further analysis. This resulted in 10,130 variants forming 14,908 instances of LD $\left(r^{2} \geq 0.6\right)$ with 345 asthma-associated variants (Additional file 1: Table S4). These 10,130 variants from the 1000GP in LD with one or more asthma variants are hereafter referred to as the ' $\mathrm{LD}$ variants' and the workflow to generate and annotate them is summarized in Fig. 2. Among the LD variants, 9147 are SNPs, 974 are insertions or deletions, and 9 are structural variants (Additional file 1: Table S5).

\section{Identification of deleterious and regulatory variants}

Many of the LD variants are predicted to have functional consequences on resulting protein structure or to impact regulation of gene expression. Table 1 reports $6 \mathrm{LD}$

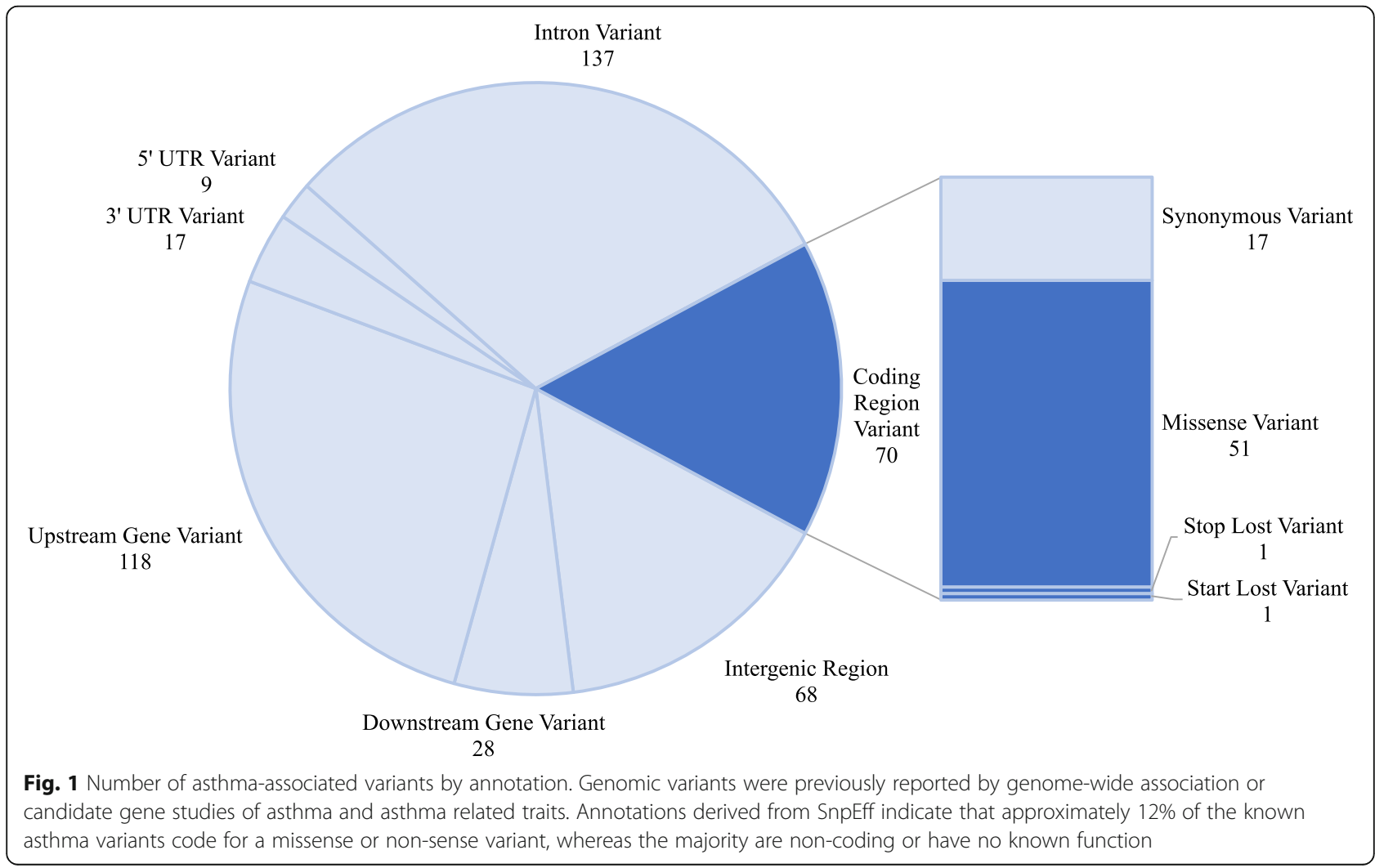




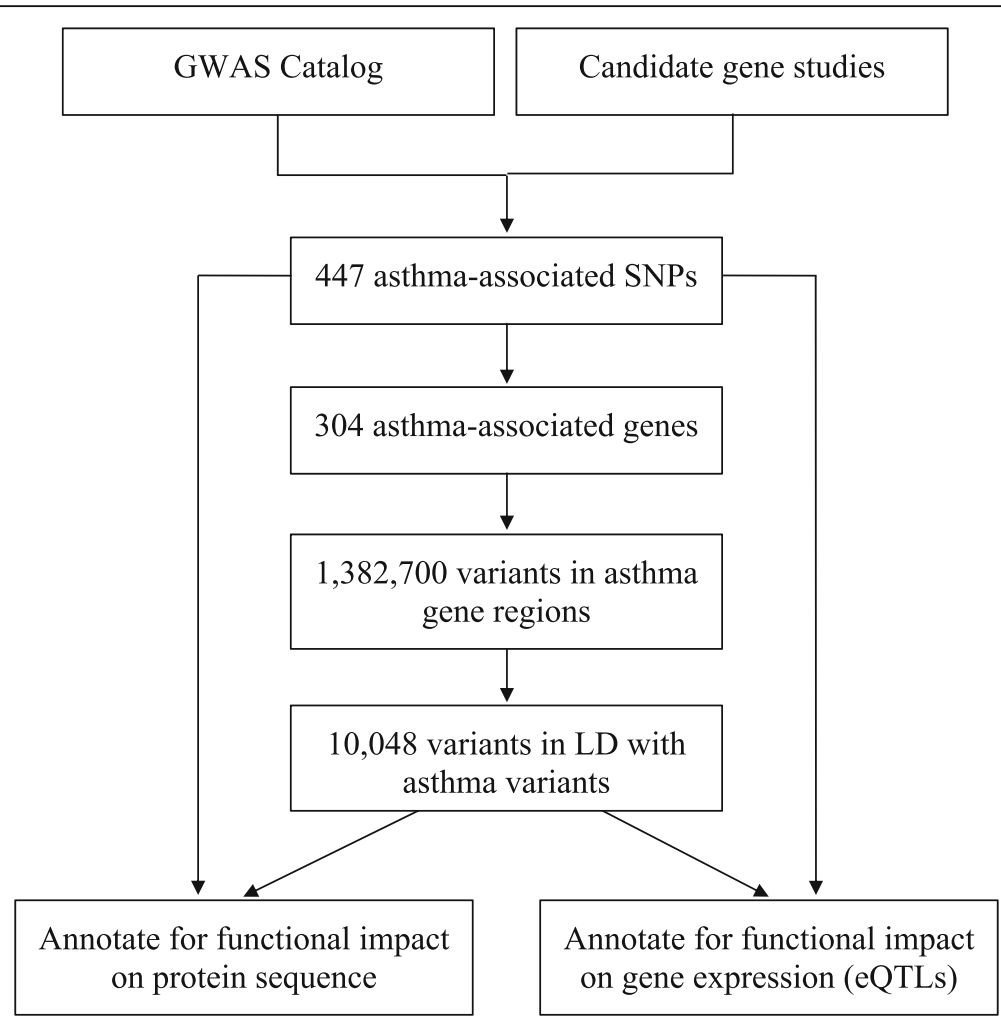

Fig. 2 Workflow for discovery of potentially causal variants in known asthma loci. First, we compiled and annotated asthma-associated SNPs from previous GWAS and candidate gene studies of asthma. We then determined the genomic coordinates of the 305 genes and extracted all sequence variations within these loci using data from the 1000 Genomes Project (1000GP). Finally, we identified and annotated those variants in linkage disequilibrium (LD) with known asthma loci to determine which may contribute to changes in protein sequence or regulate gene expression (i.e. expression quantitative trait loci or eQTL)

variants that are predicted to result in frameshift, stop lost, or splice site mutations by both genomic annotation tools SnpEff and VEP. Two deletions (rs199503730 and rs67841474) are annotated as frameshift variants, both impacting the final coding exon of major histocompatibility complex (MHC) Class I gene MICA. The deletion variant rs146576636 in the final coding exon of ADAM33 is also annotated as a frameshift variant. Two SNPs (rs8084 and rs11078928) are annotated as splice acceptor variants in $H L A-D R A$ and GSDMB respectively. Finally, the SNP rs15895 is annotated as a stop lost variant in OAS2. These variants are in $\mathrm{LD}$ with previously reported asthma variants that are less likely to be functional, such as intron variants, upstream/downstream variants, or synonymous variants.

In addition to these potentially deleterious coding variants, we identified $91 \mathrm{LD}$ variants that are predicted to be missense variants by both SnpEff and VEP. Of these, 34 are classified as deleterious and/or probably damaging by the pathogenicity prediction tools SIFT and Polyphen-2 (Table 2). The remaining 57 missense LD variants were not identified as deleterious or probably damaging using these software (Additional file 1: Table S6).
Finally, we identified LD variants that have been associated with gene expression (i.e. expression quantitative trait loci (eQTL)) using RegulomeDB. We limited our investigation to eQTLs located both at a transcription factor binding site and a DNase peak, two factors characteristic of causal eQTLs [31]. In total, we determined that 24 of the LD variants (Table 3) and 7 of the asthma-associated variants (Additional file 1: Table S7) are known eQTLs.

\section{Discussion}

In this study, we compiled a comprehensive list of asthma-associated variants from earlier candidate gene studies and GWAS in order to conduct a systematic search for causal variants that likely contribute to asthma heritability. Whole genome sequencing data from the 1000GP and functional annotation software tools were used to identify potentially deleterious variants in LD with the asthma-associated variants that could account for the original association signals at these loci. We identified variants annotated to be frameshift, splice site, stop-lost, missense, or eQTLs that are in LD with known asthma variants. The majority of these functional variants were in LD with 
Table 1 Deleterious coding variants in LD with asthma-associated SNPS

\begin{tabular}{|c|c|c|c|c|c|c|c|c|c|c|}
\hline \multicolumn{3}{|l|}{ LD Variant } & \multicolumn{3}{|c|}{ Asthma Variant } & \multicolumn{5}{|c|}{ LD r2 value ${ }^{a}$} \\
\hline ID & Gene & Annotation & $\mathrm{ID}$ & Gene(s) & Annotation & AFR & AMR & EAS & EUR & SAS \\
\hline rs 8084 & HLA-DRA & splice acceptor & rs3129890 & HLA-DRA & downstream & 0.889 & $<0.6$ & 0.815 & $<0.6$ & $<0.6$ \\
\hline rs199503730 & MICA & frameshift & rs361525 & TNF, LTA & upstream & $<0.6$ & $<0.6$ & $<0.6$ & $<0.6$ & 0.726 \\
\hline rs67841474 & MICA & frameshift & rs2428494 & $H L A-B$ & upstream & $<0.6$ & $<0.6$ & 0.671 & $<0.6$ & $<0.6$ \\
\hline rs15895 & OAS2 & stop lost & rs1293767 & OAS2 & missense & 0.749 & 0.609 & $<0.6$ & $<0.6$ & $<0.6$ \\
\hline \multirow[t]{10}{*}{ rs11078928 } & GSDMB & splice acceptor & rs907092 & IKZF3 & synonymous & $<0.6$ & 0.889 & 0.845 & 0.892 & 0.966 \\
\hline & & & rs4795397 & IKZF3 & upstream & $<0.6$ & 0.913 & 0.995 & 0.950 & 0.846 \\
\hline & & & rs11655198 & ZPBP2 & intron & $<0.6$ & 0.804 & 0.995 & 0.832 & 0.898 \\
\hline & & & rs2305480 & GSDMB & missense & 0.961 & 0.988 & 1 & 0.996 & 1 \\
\hline & & & rs62067034 & GSDMB & intron & 0.707 & 0.871 & 1 & 0.849 & 0.917 \\
\hline & & & rs1 1078927 & GSDMB & intron & 0.984 & 0.994 & 1 & 1 & 0.995 \\
\hline & & & rs2290400 & GSDMB & upstream & $<0.6$ & 0.726 & 0.945 & 0.811 & 0.704 \\
\hline & & & rs7216389 & GSDMB & upstream & $<0.6$ & 0.831 & 0.967 & 0.792 & 0.723 \\
\hline & & & rs4794820 & ORMDL3, LRRC3C & downstream & $<0.6$ & 0.688 & 0.899 & 0.731 & 0.678 \\
\hline & & & rs12450323 & IKZF3 & intron & $<0.6$ & $<0.6$ & 0.684 & $<0.6$ & $<0.6$ \\
\hline \multirow[t]{3}{*}{ rs146576636 } & ADAM33 & frameshift & rs543749 & ADAM33 & intron & $<0.6$ & $<0.6$ & 0.804 & $<0.6$ & $<0.6$ \\
\hline & & & rs574174 & ADAM33 & intron & $<0.6$ & $<0.6$ & 0.846 & $<0.6$ & $<0.6$ \\
\hline & & & rs612709 & ADAM33 & upstream & $<0.6$ & $<0.6$ & 0.800 & $<0.6$ & $<0.6$ \\
\hline
\end{tabular}

Definition of abbreviations: AFR African population; AMR American population; EAS East Asian population; EUR European population; LD Linkage disequilibrium; SAS South Asian population

${ }^{a}$ Calculated using 1000 Genomes Project Phase 3 data

asthma variants that are non-coding and have no known effects on gene transcription. In summary, we have identified numerous functional variants that could be the elusive causal variants within known asthma-associated loci and improve our understanding of the underlying mechanisms of asthma.

Several of the potentially causal variants identified in this study are found in the MHC class II locus, within the human leukocyte antigen (HLA) super-locus of the $6 \mathrm{p} 21$ chromosomal region. MHC class II genes code for proteins that play a central role in the immune system by presenting peptides to CD4+ T cells [32]. The MHC class II locus has been repeatedly associated to asthma and other immune related diseases, but finding causal variants at the locus has proven to be difficult due to its highly polymorphic nature and strong linkage disequilibrium in the region [33, 34]. We compiled 10 asthma risk variants (rs9268516, rs9272346, rs9273349, rs9273373, rs9 275698, rs9500927, rs987870, rs3104367, rs3129890, rs7775228) found at this locus from seven GWAS [35-41]. Only two of these asthma-associated variants (rs9272346 and rs7775228) have been reported to regulate gene expression (eQTLs), whereas the remainder are not known to have any functional impact on the resultant transcripts or proteins. The present study identified numerous LD variants within the same loci as these asthma variants including a spliceacceptor variant in the $H L A-D R A$ gene (rs8084); missense variants in $H L A-D Q A 1, H L A-D Q B 1, H L A-D R B$, HLADRB5, and HLA-DPA1; and several eQTLs (rs20 4992, rs3131294, rs6928482, rs1063355, rs2076523, rs2213585) that are linked to the expression of one or more MHC class II genes. Taken together these LD variants, which are more likely to have functional or even detrimental consequences, could account for the consistent associations between the MHC class II locus and asthma outcomes.

A number of the variants identified in this study are located within the 17q12-21 chromosomal region, which is the most reproducible asthma locus $[42,43]$. This genomic region consists of a number of genes, including ORMDL3, GSDMB, IKZF3, ZPBP2, and GSDMA that are in LD and may either be individually or jointly responsible for the asthma association [42]. The current study compiled 13 asthma-associated variants from the 17q12-21 region (rs3894194, rs4795397, rs62067034, rs7212938, rs7216389, rs11078927, rs2290400, rs2305480, rs4794820, rs65 03525, rs11655198, rs12450323, rs907092) that were identified across 11 independent GWAS [37-39, 4350]. Most of these variants are non-coding, save three 
Table 2 Missense variants in LD with asthma-associated variants

\begin{tabular}{|c|c|c|c|}
\hline \multicolumn{3}{|l|}{ LD Variants } & \multirow{2}{*}{$\begin{array}{l}\text { Asthma Variants } \\
I D(s)\end{array}$} \\
\hline$\overline{I D}$ & Gene & Annotation & \\
\hline rs115676129 & $A B / 3 B P$ & missense $e^{a, b}$ & rs9823506 \\
\hline rs2275254 & CHIA & missense $e^{a, b}$ & rs2282290 \\
\hline rs2305479 & GSDMB & missense $^{\mathrm{a}, \mathrm{b}}$ & $\begin{array}{l}\text { rs2305480, rs62067034, } \\
\text { rs11078927, rs7216389, } \\
\text { rs907092, rs4795397, } \\
\text { rs11655198, rs2290400, } \\
\text { rs12450323, rs4794820 }\end{array}$ \\
\hline rs1129808 & HLA-DQA1 & missense $e^{a, b}$ & rs3104367, rs9272346, rs9273373 \\
\hline rs1142332 & HLA-DQA1 & missense $e^{a, b}$ & rs3104367, rs9272346, rs9273373 \\
\hline rs1049057 & HLA-DQB1 & missense $^{a, b}$ & rs3104367, rs9272346, rs9273373 \\
\hline rs1140319 & HLA-DQB1 & missense $e^{a, b}$ & rs3104367, rs9272346, rs9273373 \\
\hline rs73022563 & PLEKHGAB & missense $e^{a, b}$ & rs62344088 \\
\hline rs6482626 & PTCHD3 & missense $e^{a, b}$ & rs660498 \\
\hline s2305089 & TBXT & missense $^{a, b}$ & rs6456042 \\
\hline rs41273547 & $A B \mid 3 B P$ & missense $^{a}$ & rs9823506 \\
\hline rs148714608 & CLSTN2 & missense $^{a}$ & rs77960860 \\
\hline rs1042308 & HLA-DPA1 & missense $^{a}$ & rs987870 \\
\hline rs1071630 & HLA-DQA1 & missense $^{a}$ & rs3104367, rs9272346, rs9273373 \\
\hline rs1129740 & HLA-DQA1 & missense $^{a}$ & rs3104367, rs9272346, rs9273373 \\
\hline rs1142328 & HLA-DQA1 & missense $^{a}$ & rs3104367, rs9272346, rs9273373 \\
\hline rs1142331 & HLA-DQA1 & missense $^{a}$ & rs3104367, rs9272346, rs9273373 \\
\hline rs3208105 & HLA-DQA1 & missense $^{a}$ & rs3104367, rs9272346, rs9273373 \\
\hline rs1049163 & HLA-DQB1 & missense $^{a}$ & rs9273373 \\
\hline rs1130398 & HLA-DQB1 & missense $^{a}$ & rs3104367, rs9272346, rs9273373 \\
\hline rs1140318 & HLA-DQB1 & missense $^{a}$ & rs3104367, rs9272346, rs9273373 \\
\hline rs1140320 & HLA-DQB1 & missense $^{a}$ & rs3104367, rs9272346, rs9273373 \\
\hline rs41558312 & MICA & missense $\mathrm{a}^{\mathrm{a}}$ & rs361525 \\
\hline rs1801280 & NAT2 & missense $^{a}$ & rs1799929, rs1208 \\
\hline rs2363468 & PIKFYVE & missense ${ }^{a}$ & rs4673397 \\
\hline rs13436090 & PLEKHG4B & missense $^{a}$ & rs62344088 \\
\hline rs3777134 & SPINK5 & missense $^{a}$ & rs2303064 \\
\hline rs682632 & TEK & missense $^{a}$ & rs72721168 \\
\hline rs1140404 & $H L A-B$ & missense $^{b}$ & rs1800629 \\
\hline rs41543121 & $H L A-B$ & missense $^{b}$ & rs1800629 \\
\hline 56417 & $H L A-B$ & missense $^{b}$ & rs361525 \\
\hline rs4193 & HLA-DQA1 & missense $^{b}$ & rs3104367, rs9272346, rs9273373 \\
\hline rs2233953 & PSORSIC2 & missense $^{b}$ & rs1800629 \\
\hline rs7686508 & SEC31A & missense $^{b}$ & rs 10022260 \\
\hline
\end{tabular}

Definition of abbreviations: $L D$ Linkage disequilibrium

${ }^{\text {aP }}$ redicted to be deleterious by Sorting Intolerant From Tolerant (SIFT)

bPredicted to be probably damaging by Polyphen-2

missense mutations: rs3894194 and rs7212938 from GSDMA and rs2305480 from GSDMB. This study identified four additional coding variants from $G S$ $D M A$ (rs56030650), GSDMB (rs2305479, rs11078928) and ZPBP2 (rs11557467) and three eQTLs for ORMDL3 (rs12946510, rs11557466, rs8076131), which are in LD with asthma variants. Notably the LD variant rs11078928 has been functionally characterized as a splice variant that removes an exon, influences transcription levels, and abolishes the biochemical activity of $G S D M B[51,52]$. This variant has been previously discussed as a possible causal variant in asthma [42, 53] and its LD with multiple asthma-associated variants supports such a hypothesis. The LD variants identified in the $17 q 12-21$ locus demonstrate the possibility that these could represent the causal variants underlying asthma risk.

While we report potentially causal variants in known asthma loci, our study also has some important limitations. For example, given that LD calculations are dependent on allele frequencies and that the majority of known asthma variants are common (minor allele frequency or MAF $>0.05$ ), our study is biased toward identifying LD variants with higher MAF [54]. Additionally, our list of asthma-associated variants from earlier GWAS and candidate gene studies are often identified from a single population and may contain some false positive or population specific results. Further studies are needed to validate these associations in additional asthma populations. Finally, we relied on annotation data from prediction tools such as SnpEff and VEP, which are limited and do not replace experimental evidence. Further studies are needed to identify and ultimately validate the true function, if any, of the LD variants identified in this study to be coding or regulatory. These additional studies include genotyping of these potentially functional variants (identified in LD with the associated variants) in asthma populations and testing them directly for correlation with asthma outcomes. Moreover, functional validation experiments are needed to confirm the biological impact of these variants on the resultant RNA transcripts or proteins, which depends on the predicted impact of the variants identified. For example, variants of high impact (Table 1) including frameshift, splice variants and premature stop codons, could be validated through direct sequencing of transcripts and mass spectrometry to detect truncated and mis-folded proteins.

\section{Conclusions}

The current study is proof of concept that previously correlated loci for asthma may tag causal variants that are in LD, which can be identified using direct sequencing data. While genetic studies of asthma to date have successfully identified hundreds of asthma loci, our understanding of the underlying mechanisms of asthma remains limited. In addition, these loci do not account 
Table 3 Regulatory variants (eQTLs) in LD with asthma-associated variants

\begin{tabular}{|c|c|c|c|c|}
\hline$\overline{\mathrm{eQTL}}$ & Affected Gene(s) & Tissue & Affected Binding Motif(s) & Asthma Variant(s) in LD \\
\hline rs14078 & NPPA & Lymphoblastoid & C/EBP & rs5065 \\
\hline rs17032120 & UNC50 & Monocytes & Whn & rs2278206 \\
\hline rs3769737 & UNC50 & Monocytes & Gm12892, Hsmm, Hsmmt, Huh7 & rs2278206 \\
\hline rs17446058 & UNC50 & Monocytes & deltaEF1 & rs2278206 \\
\hline rs5743563 & TLR10, TLR1, TLR6 & Lymphoblastoid & YY1 & rs4129009, rs4833095 \\
\hline rs5743565 & $T L R 10, T L R 6, T L R 1$ & Lymphoblastoid & GATA-4 & rs4129009, rs4833095 \\
\hline rs7941648 & RPS6KB2 & Lymphoblastoid & $\begin{array}{l}\text { CDP, BCL6, Foxd3, Sox7, Sox18, Sry, } \\
\text { Sox5 }\end{array}$ & rs1695, rs6591255 \\
\hline rs12654778 & ADRB2 & Lymphoblastoid & TP53 & rs1042713 \\
\hline rs10039559 & RAD50 & Lymphoblastoid & C/EBP & rs11745587 \\
\hline rs204992 & HLA-DQA1 & Lymphoblastoid & CAC-bindingprotein & rs204993 \\
\hline rs3131294 & $\begin{array}{l}\text { HLA-DQA1, HLA-C, HLA-DQB1, } \\
\text { HLA-DRB1, HLA-DRB5, HLA-DRA, } \\
E R G^{a}, H C G 27, H L A-C, L I M S 1^{a}\end{array}$ & $\begin{array}{l}\text { Lymphoblastoid, } \\
\text { Monocytes }\end{array}$ & AIRE & rs3135388 \\
\hline rs6928482 & HLA-DQA1, HLA-DQA2 & Lymphoblastoid & Zfp410 & rs3104367, rs9272346, rs9273373 \\
\hline rs1063355 & $\begin{array}{l}\text { HLA-DQA1, HLA-DQB1, HLA-DRB1, } \\
\text { HLA-DRA }\end{array}$ & Lymphoblastoid & Isgf3g & rs3104367, rs9272346, rs9273373 \\
\hline rs2076523 & $\begin{array}{l}\text { HLA-DQA2, HLA-DQB1, HLA- } \\
\text { DQA2 }\end{array}$ & Lymphoblastoid & Hepg2 & rs9268516 \\
\hline rs2213585 & $\begin{array}{l}\text { HLA-DQB1, HLA-DRB1, HLA-DRB5, } \\
\text { HLA-DRA, HLA-DQA1 }\end{array}$ & Lymphoblastoid & Zfp410 & rs3129890 \\
\hline rs6906021 & POLRZA, TAF1, PAX5 & Lymphoblastoid & Pit-1 & rs3104367, rs9272346, rs9273373 \\
\hline rs7970524 & $H A L, L T A 4 H$ & Monocytes & $A R$ & rs2540493 \\
\hline rs12429692 & ALOX5AP & Monocytes & PU.1 & rs10507391 \\
\hline rs3862469 & DEXI, LOC642755 & Monocytes & NF-E2, ZBRK1, MAFB & rs9923856 \\
\hline rs11642631 & DEXI, LOC642755 & Monocytes & VDR & rs9923856 \\
\hline rs12946510 & $\begin{array}{l}\text { KRT222P, MEd24, NR1D1, } \\
\text { ORMDL3 }\end{array}$ & Lymphoblastoid & FOXO1, IRF1, Srf, Elf3 & $\begin{array}{l}\text { rs } 907092, \text { rs4795397, rs } 11655198, \\
\text { rs2305480, rs62067034, rs11078927, } \\
\text { rs2290400, rs7216389, rs4794820, } \\
\text { rs12450323 }\end{array}$ \\
\hline rs8076131 & KRT222P, NR1D1, ORMDL3 & Lymphoblastoid & Tcfe2a & $\begin{array}{l}\text { rs } 2305480, \text { rs } 11078927, \text { rs } 7216389, \\
\text { rs907092, rs4795397, rs 11655198, } \\
\text { rs62067034, rs2290400, rs4794820, } \\
\text { rs } 12450323\end{array}$ \\
\hline rs11557466 & ORMDL3 & Lymphoblastoid & HOXA5, REST, NRSE, HIF1 & $\begin{array}{l}\text { rs2305480, rs62067034, rs11078927, } \\
\text { rs907092, rs4795397, rs11655198, } \\
\text { rs2290400, rs7216389, rs4794820, } \\
\text { rs12450323 }\end{array}$ \\
\hline rs8069732 & TBKBP1 & Monocytes & $\begin{array}{l}\text { NF-E2, Fos, GCN4, hoxa9, Cdx-2, } \\
\text { Hoxa11, Hoxc10, Hoxc11, Hoxc9, } \\
\text { Hoxa9, Hoxb9 }\end{array}$ & rs5918 \\
\hline
\end{tabular}

Definition of abbreviations: $e Q T L$ expression quantitative trait locus; $L D$ linkage disequilibrium

${ }^{\text {aTrans }}$ eQTL effect

for all of the asthma heritability and have limited clinical applications due to the fact that the majority of associated variants do not represent the true causative loci. Identification of these causal variants underlying the genetic associations will be instrumental in improving our understanding of the underlying mechanisms of asthma. Ultimately, knowledge of the casual variants will help to facilitate the development of more accurate clinical tests for determining risk and treatment options for asthma.

\section{Additional file}

Additional file 1: Table S1. Asthma-associated SNPs from genomewide association studies. Table S2. Asthma-associated SNPs from candidate gene studies. Table S3. Asthma-associated variants with protein 
coding annotation. Table S4. Ethnic specific linkage disequilibrium between asthma variants and 1000GP variants. Table S5. Annotation of all variants in linkage disequilibrium with asthma variants. Table S6. Missense variants not predicted to be deleterious in linkage disequilibrium with asthma-associated variants. Table S7. eOTLs within asthmaassociated variants. (XLSX $1149 \mathrm{~kb})$

\section{Abbreviations}

1000GP: 1000 Genomes Project; eQTL: Expression quantitative trait loci; GWAS: Genome-wide association study; HLA: Human leukocyte antigen; LD: Linkage disequilibrium; MHC: Major histocompatibility complex; NHGRIEBI: National Human Genome Research Institute-European Bioinformatics Institute; SIFT: Sorting Intolerant From Tolerant; SNP: Single nucleotide polymorphism; VEP: Variant Effect Predictor

\section{Acknowledgements}

The authors thank the Centre for Advanced Computing (CAC) at Queen's University in Kingston, Ontario for computational resources and support. The CAC is funded by: the Canada Foundation for Innovation, the Government of Ontario, and Queen's University.

\section{Funding}

$J C$ and QLD receive funding from the Canadian Institutes of Health Research and Queen's University.

\section{Availability of data and material}

The 1000 Genomes Project Phase 3 dataset used for the current analyses is publicly available from The International Genome Sample Resource (http:// www.internationalgenome.org/).

\section{Authors' contributions}

Conception and design: QLD. Data analysis: MN, JL, JC. Wrote manuscript: MN, JC, QLD. Critical revision of the manuscript: all authors. All authors read and approved the final manuscript.

\section{Ethics approval and consent to participate}

Not applicable.

\section{Consent for publication}

Not applicable.

\section{Competing interests}

The authors declare that they have no competing interests.

\section{Publisher's Note}

Springer Nature remains neutral with regard to jurisdictional claims in published maps and institutional affiliations.

Received: 12 August 2018 Accepted: 23 November 2018

Published online: 12 December 2018

\section{References}

1. Vos T, Flaxman AD, Naghavi M, et al. Years lived with disability (YLDs) for 1160 sequelae of 289 diseases and injuries 1990-2010: a systematic analysis for the global burden of disease study 2010. Lancet. 2012;380:2163-96. https://doi.org/10.1016/S0140-6736(12)61729-2.

2. Masoli M, Fabian D, Holt S, et al. The global burden of asthma: executive summary of the GINA dissemination committee report. Allergy. 2004;59: 469-78. https://doi.org/10.1111/j.1398-9995.2004.00526.x.

3. Duffy DL, Martin NG, Battistutta D, et al. Genetics of asthma and Hay fever in Australian twins. Am Rev Respir Dis. 1990;142:1351-8. https://doi.org/10. 1164/ajrccm/142.6_Pt_1.1351.

4. Nieminen MM, Kaprio J, Koskenvuo M. A population-based study of bronchial asthma in adult twin pairs. Chest. 1991;100:70-5. https://doi.org/ 10.1378/chest.100.1.70. Accessed 21 Dec 2017.

5. Thomsen SF, Van Der Sluis S, Kyvik KO, et al. Estimates of asthma heritability in a large twin sample Clinical \&amp; Experimental Allergy. Clin Exp Allergy. 2010:1054-61. https://doi.org/10.1111/j.1365-2222.2010.03525.x.
6. Vicente $\mathrm{CT}$, Revez JA, Ferreira MAR. Lessons from ten years of genome-wide association studies of asthma. Clin Transl Immunol. 2017;6:e165. https://doi. org/10.1038/cti.2017.54.

7. Ober C, Yao T-C. The genetics of asthma and allergic disease: a 21st century perspective. Immunol Rev. 2011;242:10-30. https://doi.org/10.1111/j.1600065X.2011.01029.x

8. Manolio TA, Collins FS, Cox NJ, et al. Finding the missing heritability of complex diseases. Nature. 2009;461:747-53. https://doi.org/10.1038/ nature08494.

9. Hindorff LA, Sethupathy P, Junkins HA, et al. Potential etiologic and functional implications of genome-wide association loci for human diseases and traits. Proc Natl Acad Sci U S A. 2009;106:9362-7. https://doi.org/10. 1073/pnas.0903103106.

10. MacArthur DG, Manolio TA, Dimmock DP, et al. Guidelines for investigating causality of sequence variants in human disease. Nature. 2014;508:469-76. https://doi.org/10.1038/nature13127.

11. Pritchard JK, Przeworski M. Linkage disequilibrium in humans: models and data. Am J Hum Genet. 2001;69:1-14. https://doi.org/10.1086/321275.

12. Auton $A$, Abecasis $G R$, Altshuler $D M$, et al. A global reference for human genetic variation. Nature. 2015;526:68-74. https://doi.org/10.1038/ nature15393.

13. MacArthur J, Bowler E, Cerezo M, et al. The new NHGRI-EBI catalog of published genome-wide association studies (GWAS catalog). Nucleic Acids Res. 2017;45:D896-901. https://doi.org/10.1093/nar/gkw1133.

14. Melén E, Kho AT, Sharma $\mathrm{S}$, et al. Expression analysis of asthma candidate genes during human and murine lung development. Respir Res. 2011;12:86. https://doi.org/10.1186/1465-9921-12-86.

15. Sharma A, Menche J, Huang CC, et al. A disease module in the interactome explains disease heterogeneity, drug response and captures novel pathways and genes in asthma. Hum Mol Genet. 2015;24:3005-20. https://doi.org/10. 1093/hmg/ddv001.

16. Vercelli D. Discovering susceptibility genes for asthma and allergy. Nat Rev Immunol. 2008;8:169-82. https://doi.org/10.1038/nri2257.

17. Weiss ST, Raby BA, Rogers A. Asthma genetics and genomics 2009. Curr Opin Genet Dev. 2009;19:279-82. https://doi.org/10.1016/j.gde.2009.05.001.

18. Pruitt KD, Brown GR, Hiatt SM, et al. RefSeq: an update on mammalian reference sequences. Nucleic Acids Res. 2014;42:D756-63. https://doi.org/10. 1093/nar/gkt1114.

19. Karolchik D, Hinrichs AS, Furey TS, et al. The UCSC table browser data retrieval tool. Nucleic Acids Res. 2004;32:493D-496. https://doi.org/10.1093/ nar/gkh103.

20. Chang CC, Chow CC, Tellier LC, et al. Second-generation PLINK: rising to the challenge of larger and richer datasets. Gigascience. 2015;4:7. https://doi. org/10.1186/s13742-015-0047-8.

21. Cingolani P, Platts A, Wang $L L$, et al. A program for annotating and predicting the effects of single nucleotide polymorphisms, SnpEff: SNPs in the genome of Drosophila melanogaster strain w1118; iso-2; iso-3. Fly (Austin). 2012;6:80-92. https://doi.org/10.4161/fly.19695.

22. McLaren W, Gil L, Hunt SE, et al. The Ensembl variant effect predictor. Genome Biol. 2016;17:122. https://doi.org/10.1186/s13059-016-0974-4.

23. Kumar P, Henikoff S, Ng PC. Predicting the effects of coding nonsynonymous variants on protein function using the SIFT algorithm. Nat Protoc. 2009:4:1073-81. https://doi.org/10.1038/nprot.2009.86.

24. Adzhubei IA, Schmidt S, Peshkin L, et al. A method and server for predicting damaging missense mutations. Nat Methods. 2010;7:248-9. https://doi.org/ 10.1038/nmeth0410-248.

25. Lappalainen I, Lopez J, Skipper L, et al. DbVar and DGVa: public archives for genomic structural variation. Nucleic Acids Res. 2013;41:D936-41. https:// doi.org/10.1093/nar/gks1213.

26. Boyle AP, Hong EL, Hariharan M, et al. Annotation of functional variation in personal genomes using RegulomeDB. Genome Res. 2012;22:1790-7. https://doi.org/10.1101/gr.137323.112.

27. ENCODE Project Consortium TEP. An integrated encyclopedia of DNA elements in the human genome. Nature. 2012;489:57-74. https://doi.org/10. 1038/nature11247

28. Barrett T, Wilhite $S E$, Ledoux $P$, et al. NCBI GEO: archive for functional genomics data sets-update. Nucleic Acids Res. 2012;41:D991-5. https://doi. org/10.1093/nar/gks1193.

29. Charles BA, Shriner D, Rotimi CN. Accounting for linkage disequilibrium in association analysis of diverse populations. Genet Epidemiol. 2014;38:26573. https://doi.org/10.1002/gepi.21788. 
30. Ong RTH, Teo YY. varLD: a program for quantifying variation in linkage disequilibrium patterns between populations. Bioinformatics. 2010;26:126970. https://doi.org/10.1093/bioinformatics/btq125.

31. Albert FW, Kruglyak $\mathrm{L}$. The role of regulatory variation in complex traits and disease. Nat Rev Genet. 2015;16:197-212. https://doi.org/10.1038/nrg3891.

32. Watts $C$. The exogenous pathway for antigen presentation on major histocompatibility complex class II and CD1 molecules. Nat Immunol. 2004; 5:685-92. https://doi.org/10.1038/ni1088.

33. Kontakioti E, Domvri K, Papakosta D, et al. HLA and asthma phenotypes/ endotypes: a review. Hum Immunol. 2014;75:930-9. https://doi.org/10.1016/ J.HUMIMM.2014.06.022.

34. Handunnetthi L, Ramagopalan SV, Ebers GC, et al. Regulation of major histocompatibility complex class II gene expression, genetic variation and disease. Genes Immun. 2010;11:99-112. https://doi.org/10.1038/gene.2009.83.

35. Ramasamy A, Kuokkanen M, Vedantam S, et al. Genome-wide association studies of asthma in population-based cohorts confirm known and suggested loci and identify an additional association near HLA. PLoS One 2012;7:e44008. https://doi.org/10.1371/journal.pone.0044008.

36. Lasky-Su J, Himes BE, Raby BA, et al. HLA-DQ strikes again: genome-wide association study further confirms HLA-DQ in the diagnosis of asthma among adults. Clin Exp Allergy. 2012;42:1724-33. https://doi.org/10.1111/cea.12000.

37. Moffatt MF, Gut IG, Demenais F, et al. A large-scale, consortium-based genomewide association study of asthma. N Engl J Med. 2010;363:1211-21. https://doi.org/10.1056/NEJMoa0906312.

38. Pickrell JK, Berisa T, Liu JZ, et al. Detection and interpretation of shared genetic influences on 42 human traits. Nat Genet. 2016;48:709-17. https:// doi.org/10.1038/ng.3570.

39. Ferreira MAR, Matheson MC, Tang CS, et al. Genome-wide association analysis identifies 11 risk variants associated with the asthma with hay fever phenotype. J Allergy Clin Immunol. 2014;133:1564-71. https://doi.org/10. 1016/j.jaci.2013.10.030

40. Noguchi E, Sakamoto H, Hirota T, et al. Genome-wide association study identifies HLA-DP as a susceptibility gene for pediatric asthma in Asian populations. PLoS Genet. 2011;7:e1002170. https://doi.org/10.1371/journal. pgen. 1002170

41. Hirota T, Takahashi A, Kubo M, et al. Genome-wide association study identifies three new susceptibility loci for adult asthma in the Japanese population. Nat Genet. 2011;43:893-6. https://doi.org/10.1038/ng.887.

42. Stein MM, Thompson EE, Schoettler N, et al. A decade of research on the 17q1221 asthma locus: Piecing together the puzzle. J Allergy Clin Immunol Published Online First: 4 January. 2018. https://doi.org/10.1016/J.JACl.2017.12.974.

43. Torgerson DG, Ampleford EJ, Chiu GY, et al. Meta-analysis of genome-wide association studies of asthma in ethnically diverse north American populations. Nat Genet. 2011;43:887-92. https://doi.org/10.1038/ng.888. Accessed 28 Nov 2017.

44. Wan Yl, Shrine NRG, Soler Artigas M, et al. Genome-wide association study to identify genetic determinants of severe asthma. Thorax. 2012;67:762-8. https://doi.org/10.1136/thoraxjnl-2011-201262.

45. Moffatt MF, Kabesch M, Liang $L$, et al. Genetic variants regulating ORMDL3 expression contribute to the risk of childhood asthma. Nature. 2007;448: 470-3. https://doi.org/10.1038/nature06014.

46. Yan Q, Brehm J, Pino-Yanes $M$, et al. A meta-analysis of genome-wide association studies of asthma in Puerto Ricans. Eur Respir J. 2017;49: 1601505. https://doi.org/10.1183/13993003.01505-2016.

47. Nieuwenhuis MA, Siedlinski $M$, van den Berge $M$, et al. Combining genomewide association study and lung $\mathrm{eQTL}$ analysis provides evidence for novel genes associated with asthma. Allergy. 2016;71:1712-20. https:// doi.org/10.1111/all.12990

48. Almoguera B, Vazquez $L$, Mentch F, et al. Identification of four novel loci in asthma in European American and African American populations. Am J Respir Crit Care Med. 2017;195:456-63. https://doi.org/10.1164/rccm.201604-08610C

49. Bønnelykke K, Sleiman P, Nielsen K, et al. A genome-wide association study identifies CDHR3 as a susceptibility locus for early childhood asthma with severe exacerbations. Nat Genet. 2013;46:51-5. https://doi.org/10.1038/ng.2830.

50. Ferreira MAR, McRae AF, Medland SE, et al. Association between ORMDL3, IL1RL1 and a deletion on chromosome 17q21 with asthma risk in Australia. Eur J Hum Genet. 2011;19:458-64. https://doi.org/10.1038/ejhg.2010.191.

51. Morrison FS, Locke JM, Wood AR, et al. The splice site variant rs 11078928 may be associated with a genotype-dependent alteration in expression of GSDMB transcripts. BMC Genomics. 2013;14:627. https://doi.org/10.1186/ 1471-2164-14-627
52. Panganiban RA, Sun $M$, Dahlin A, et al. A functional splice variant associated with decreased asthma risk abolishes the ability of gasdermin B to induce epithelial cell pyroptosis. J Allergy Clin Immunol Published Online First: 9 January. 2018. https://doi.org/10.1016/j.jaci.2017.11.040.

53. Igartua C, Myers RA, Mathias RA, et al. Ethnic-specific associations of rare and low-frequency DNA sequence variants with asthma. Nat Commun. 2015;6:5965. https://doi.org/10.1038/ncomms6965.

54. VanLiere JM, Rosenberg NA. Mathematical properties of the $r 2$ measure of linkage disequilibrium. Theor Popul Biol. 2008;74:130-7. https://doi.org/10. 1016/j.tpb.2008.05.006.

\section{Ready to submit your research? Choose BMC and benefit from:}

- fast, convenient online submission

- thorough peer review by experienced researchers in your field

- rapid publication on acceptance

- support for research data, including large and complex data types

- gold Open Access which fosters wider collaboration and increased citations

- maximum visibility for your research: over $100 \mathrm{M}$ website views per year

At BMC, research is always in progress.

Learn more biomedcentral.com/submissions 